\title{
Realidad Sociojurídica del Seguro Obligatorio de Accidentes de Tránsito en Neiva (Colombia)
}

Semillero de Investigación "INSOAT"

Camila Francesca Garces Prieto Danny Joan Guevara Silva Fredy Hernan López Córdoba Hector Fabián Losada Vargas Jorge Eduardo Santos Zúñiga Juan Miguel Cabrera Pedreros Tudor González García

Tutora: Dra. Diana Marcela Ortíz Tovar Grupo de Investigación Synergia Universidad Surcolombiana

Articulo recibido: 21/09/2009 Aprobado:02/11/2009

\section{RESUMEN}

En la ciudad de Neiva (Huila) se presentó durante el periodo 2005-2007 un gran número de accidentes de tránsito, en donde el seguro obligatorio de accidentes de tránsito (SOAT) desempeñó un papel importante cubriendo los gastos médicos, quirúrgicos y hospitalarios; además de incapacidades permanentes, muerte, gastos funerarios, y gastos de transporte y movilización de las víctimas en la gran mayoría de estos accidentes. Pero en otros casos, la efectividad yel desempeño del SOAT no es la esperada.

\section{ABSTRACT}

In the city of Neiva (Huila) it was presented during the period 2005-2007 a great number of traffic accidents where the obligatory insurance of traffic accidents (SOAT) it played an important part covering the medical, surgical and hospital expenses; besides permanent inabilities, death, funeral expenses, and expenses of transport and the victims' mobilization in the great majority of these accidents. But in other cases the effectiveness and the acting of the SOAT is not the prospective one.

\section{PALABRAS CLAVE}

Seguro obligatorio, accidente de tránsito, amparo, tomador, víctima, evasión, fraude, aseguradora.

\section{KEYWORDS}

Obligatory insurance, traffic accidents, abetting, taker, victim, evasion, fraud, insurance company 


\section{INTRODUCCIÓN}

La presente investigación, se orientó al estudio del SOAT (seguro obligatorio de accidentes de tránsito) que protege la vida y la integridad personal de los colombianos que resultan afectados en la ocurrencia de accidentes de tránsito, que se presenten dentro del territorio nacional; en forma de indemnización automática a favor de las víctimas sin necesidad de acreditar culpa o el factor subjetivo de las conductas 0 causantes del daño.

\section{REFERENTE TEÓRICO}

Se puede apreciar que el SOAT tuvo su origen en el Convenio Europeo sobre la Responsabilidad Civil por daños causados por vehículos automóviles firmado el 14 de mayo de 1973, en el que se comprometía a cada Estado contratante a adaptar su derecho interno a las disposiciones de tal convenio.

Respecto a la historia de la implementación del SOAT en Colombia, es evidente que solamente hasta el primero $\left(1^{\circ}\right)$ de abril de 1988 entró en operación el seguro obligatorio SOAT, a pesar de estar reglamentado desde el año 1970 por el Código Nacional de Tránsito. A partir de allí las tarifas del SOAT, así como sus condiciones generales han sido modificadas en el transcurso de losaños.

Se realizó un análisis total de la reglamentación jurídica del SOAT desde sus inicios, entre los más importantes se encuentran el Decreto Ley 1344 de 1970 por el cual se impone la obligatoriedad del seguro por daños a las personas causados en accidentes de tránsito; la Ley 33 de 1986 a partir del cual entra en operación el seguro obligatorio SOAT y el Decreto 3990 de 2007 en el cual se regula el proceso de reclamación del SOAT.

\section{PLANTEAMIENTO INVESTIGATIVO}

Cuando se implementó la obligatoriedad del seguro de accidentes, se pretendió cumplir unos objetivos determinados, objetivos que son la razón de ser de este seguro y por ende es pertinente tenerlos en cuenta, dentro de los que se encuentra la provisión de soluciones a fenómenos que afectan a grupos familiares, ya que el legislador no desconocía que la perdida de uno de sus miembros por un accidente de circulación, muchas veces los dejaban en la indigencia cuando dependían económicamente de este.

Otra de las razones, obedeció a que gran porcentaje de los recursos del sector de la salud pública eran utilizados en la atención de las víctimas de accidentes de tránsito, por lo cual era necesario convocar la participación del sector privado en la cobertura de estos gastos, a fin de evitar inestabilidad en la prestación del servicio de salud.

Pero quizás el objetivo más relevante, es el deseo de proteger a los perjudicados por un accidente de tránsito ante la eventualidad de que el responsable se encuentre insolvente; con lo anterior se hace referencia al alcance del SOAT.

Por otro lado se analizó la efectividad de este seguro, entendiendo por esta, si se está o no cumpliendo con la obligación de adquirir el SOAT, pues se puede presentar cierta renuencia por parte de los usuarios, la cual se puede manifestar en la no adquisición de este seguro o peor aún en el porte de un SOAT falso.

Finalmente se indagó por la incidencia de su utilización, bien sea positiva; como seria su papel activo en la sufragación de los gastos en razón de un accidente de tránsito, o bien negativa; como sería el incremento en el costo de vida por su adquisición.

Todo lo anterior nos llevó a formular la siguiente pregunta ¿Cuál ha sido el alcance, la efectividad e incidencia que ha tenido la aplicación del SOAT en la ciudad de Neiva en el periodo 2005-2007? 


\section{JUSTIFICACIÓN}

Colombia es un Estado Social de Derecho y por ende está obligado a velar por la protección de sus asociados. Esto se ve manifestado en el SOAT, pues éste se destina a la protección de la vida e integridad de las víctimas de un accidente de tránsito, por lo cual es innegable la función social que presta, y es precisamente este carácter social lo que destaca la relevancia de esta investigación.

Igualmente cabe resaltar que en la ciudad de Neiva se presenta un gran número de accidentes de tránsito, comparado con algunas ciudades de similar población y es donde el SOAT juega un papel importante cubriendo los gastos establecidos en los amparos del mismo.

\section{RESULTADOS}

En adelante, se presentaran los resultados teóricos referentes a los elementos, atributos y características del SOAT y seguidamente se mostrarán los resultados estadísticos y documentales de la investigación en relación con el alcance, efectividad e incidencia de la aplicación del SOAT en la ciudad de Neiva dentro del periodo 2005-2007.

\subsection{Resultados teóricos.}

En este apartado se tratarán las cuestiones generales del SOAT, para posteriormente iniciar un análisis y desarrollo con técnica y precisión del objeto concreto de la investigación.

\subsubsection{Sistemas de Seguro Obligatorio de Automóviles.}

En Latinoamérica se contemplan dos sistemas de seguro obligatorio de automóviles. Ellos son: El seguro de accidentes personales y el seguro de responsabilidad civil.

El seguro de accidentes personales participa de las características del seguro de personas y tiene como fin reparar en forma automática, los daños corporales sufridos por las víctimas de accidentes, sin importar para ello las cuestiones atinentes a la responsabilidad civil. Colombia se encuentra dentro de este sistema.

En lo que al seguro de responsabilidad civil se refiere, se debe decir que se fundamenta en un seguro de daños y está llamado a operar cuando al asegurado conforme a la ley civil se le considere responsable del daño. En otros términos, en esta clase de seguro, el asegurador se encuentra obligado a indemnizar los perjuicios patrimoniales que cause el asegurado con motivo de la responsabilidad en que incurra.

\subsubsection{Definición delSOAT.}

El SOAT, según el Estatuto Orgánico Del Sistema Financiero, "es el seguro obligatorio de accidentes de tránsito que deben adquirir todos los vehículos que circulen por el territorio nacional". (Articulo 192)

En caso de la ocurrencia de un accidente de tránsito, el SOAT es quien va a cubrir los daños corporales que hayan sufrido las personas, ya sean peatones, conductores o pasajeros. Por eso es necesario determinar que dichos daños han sido sufridos por las personas, y no por los vehículos como consecuencia de la colisión.

\subsubsection{Amparos delSOAT.}

Los amparos nombrados en su regulación normativa son los siguientes:

- Primer amparo: Garantiza los recursos necesarios para brindar la adecuada atención médica inmediata que requieren todas las personas lesionadas.

- Segundo amparo: Se refiere a la indemnización por incapacidad permanente.

- Tercer amparo: Se trata de la indemnización por muerte de la víctima ocurrida al momento del accidente de tránsito, o en el año siguiente al siniestro.

- Cuarto amparo: Relacionado con los gastos funerarios de quienes fallecen.

- Quinto amparo: Contiene los gastos de 
transporte y movilización de las víctimas al centro asistencial.

\subsection{Resultados Estadísticos y documentales.}

En este punto se hará referencia al alcance, efectividad e incidencia del SOAT con apoyo de la doctrina, la jurisprudencia, datos estadísticos, casuística, entre otros, tratando de concretarlo en la medida de lo posible al ámbito territorial que comprende la ciudad de Neiva.

\subsubsection{Alcance}

Uno de los objetivos y quizá el más importante que pretende alcanzar el SOAT es la adecuada protección a las víctimas de un accidente de tránsito: "...no se debe permitir la circulación de vehículos sin la garantía adecuada para responder de los daños que causen, sin la solvencia necesaria para indemnizar a las víctimas. Para ello es preciso establecer el principio de obligatoriedad del seguro de automóviles". (Compilación de la Superintendencia Bancaria. (1990). Pág. 191)

En este orden de ideas, se procedió a determinar el alcance de protección a la víctima por el SOAT, desde una perspectiva jurídica. Para cumplir con ello, fue pertinente realizar el estudio a partir de tres aspectos: Los vehículos objetos de la obligación, los accidentes de tránsito y la calidad de la víctima.

El Estatuto Orgánico del Sistema Financiero en su artículo 192 numeral $1^{\circ}$, señala la obligatoriedad del seguro para todo vehículo automotor que transite por el territorio nacional. A su vez, el mencionado estatuto, define el vehículo automotor como "todo aparato provisto de un motor propulsor, destinado a circular por el suelo para el transporte de personas o bienes, incluyendo cualquier elemento montado sobre ruedas que le sea acoplado". (Artículo 192 numeral $3^{\circ}$ )

Desde este ángulo se aprecia que el campo de protección a la víctima es bastante amplio. No obstante lo anterior, existen ciertos casos, como ciertos tipos de vehículos, que no son objetos de esta obligación.
No se puede desconocer que en la praxis, cierto tipo de maquinaria, ejemplo típico el tractor, es utilizado como medio de transporte para trayectos cortos, pese a que esta no sea su función. Este tipo de maquinaria no se encuentra obligada a la adquisición de un seguro contra accidentes de tránsito, pues están destinados a actividades agrícolas y no a la circulación por el territorio nacional.

Otro caso similar es la posibilidad de expedir una póliza de seguro obligatorio de accidentes de tránsito a un remolque.

A primera vista, parece justificable la implementación de un seguro obligatorio para los anteriores casos y demás similares; sin embargo la adquisición de un seguro, implica un costo, lo cual demerita su empleo si se tiene en cuenta que los accidentes causados por esta clase de máquinas al circular por una carretera no son muy frecuentes. Además cabe anotar, que las víctimas en estos eventos no quedan desprotegidas, pues pueden buscar que se declare la responsabilidad civil del causante del daño.

Ahora, es importante determinar que eventos abarca la noción de accidentes de tránsito, pues así mismo es el campo de protección a la victima.

Conforme al Decreto 1283 de 1996, "uno de los requisitos para que un hecho pueda ser considerado como accidente de tránsito es que el daño tenga ocurrencia en una vía publica o en una privada con acceso al público y con destinación especifica al tránsito de vehículos, personas y/o animales". (Articulo 30)

Es evidente que se restringe en cierta medida la protección a la víctima. Para su examen, mencionaremos algunos casos sometidos a consideración del comité jurídico de FASECOLDA, los cuales confirma la mencionada restricción.

En uno de estos casos, se considera la posibilidad de ser objeto de cobertura por el SOAT la lesión que sufrió una persona que se encontraba en el parqueadero de un edificio, al cual solo tiene 
acceso a los habitantes del mismo, mientras un conductor sacaba su automóvil.

El comité jurídico de FASECOLDA al respecto, manifestó lo siguiente: "En opinión del comité se trata de un recinto privado y las lesiones en accidentes acaecidos en dichas vías, no serán catalogados como de tránsito y por lo tanto, no estarán cubiertas por el seguro". (SOAT. Conceptos del Comité Jurídico de FASECOLDA. Cuarta edición. Págs. 7-8)

No es de cuestionar la opinión del comité jurídico de FASECOLDA, pues es conforme a la ley. Sin embargo, no se puede desconocer que la ley consagra una clara restricción a la protección de la víctima, por el solo hecho que el accidente tenga ocurrencia en un lugar que no es de acceso al público en general. Este hecho genera cierta desigualdad contrario a lo pronunciado también por el comité si el accidente ocurriera en un parqueadero público, pues en ese caso, el SOAT cubriría los gastos generados por el accidente.

Todo lo anterior refleja una clara limitante al campo de protección de la víctima sin justificación alguna, pues la conducción de automóviles genera un grave riesgo social, independientemente de las características de la vía en donde se desarrolle dicha actividad.

En cuanto al alcance de protección a la víctima no existe limitación, pues el Estatuto Orgánico del Sistema Financiero dispone que "para movilizarse por el territorio nacional todo vehículo debe estar amparado por un seguro obligatorio que cubra los daños corporales que se causen a las personas en accidentes de tránsito, independientemente de su condición". (Artículo 192 numeral 10). Igualmente el mencionado estatuto, indica que "el valor de estas coberturas se entiende fijado para cada víctima". (Artículo 193 numeral 10). Todo esto nos permite señalar que el SOAT opera independientemente de la calidad de la víctima, por tanto se aplica sin importar la condición de la víctima o el numero de víctimas.
Lo anterior se refleja en una consulta realizada al comité jurídico de FASECOLDA, en la cual se pone de presente la expedición de un seguro obligatorio de accidentes de tránsito para una volqueta, la cual puede llevar tres ocupantes en la cabina. El vehículo se accidenta causando lesiones a los tres ocupantes de la cabina y a ocho mas que viajaban en el vehículo. Con base en esto se pregunta si los ocho ocupantes que viajaban en el automotor, se encuentran amparados por el SOAT.

Al respecto, el comité manifestó que "así se esté contrariando las disposiciones de tránsito, no implica que se excluya su cubrimiento debido a que la póliza cubre a todas las víctimas de accidentes de tránsito". (SOAT. Conceptos del Comité Jurídico de FASECOLDA. Cuarta edición Pág. 43)

Así las cosas, queda claro que la calidad de la víctima no reprenda un limitante al alcance de protección de la misma.

\subsubsection{Efectividad}

Para determinar la efectividad del SOAT, es preciso tener en cuenta los mecanismos con los que cuenta el Estado para lograr las metas fijadas al momento de la creación del mismo.

Indispensablemente se debe traer a colación las normas nacionales que regulan esta materia debido a que son estas las que en últimas determinan la aplicabilidad del seguro a nivel nacional y por supuesto en la ciudad Neiva.

Para evitar innumerables referencias basta con señalar que el sistema jurídico colombiano posee amplia normatividad en lo que respecta al SOAT, especial mención se le debe hacer al Estatuto Orgánico del Sistema Financiero (Capítulos IV y V, artículos 192-200) el cual establece el régimen del seguro obligatorio de daños corporales causados a las personas en accidentes de tránsito. Este se hace importante al tener en cuenta que regula todos los aspectos de la póliza, sobre todo su obligatoriedad y función social. 
Igualmente existen normas no menos importantes tales como el Código Nacional de Transito (Ley 769 del 2002) y el Decreto 1283 de 1999 (Capitulo V. Subcuenta de Seguro de Riesgos Catastróficos y Accidentes de Tránsito), entre otras, que velan por el goce efectivo del SOAT, ya sea imponiendo sanciones por no adquirirlo 0 determinando claramente los pasos a seguir para su reclamación en caso de accidente.

Por otra parte, el Estado mediante instituciones tales como la Superintendencia Nacional de Salud y la Superintendencia Financiera, vigila el estricto cumplimiento de las normas vigentes referentes al SOAT. En este sentido el Decreto 1259 de 1994 (Articulo 4) estipula como sujetos de inspección, vigilancia y control al Fondo del Seguro Obligatorio de Accidentes de Tránsito, además de estipular dentro sus funciones y facultades (Articulo 5), aspectos para la protección del seguro de accidentes de tránsito.

Por ultimo, es pertinente rescatar que para hacer efectiva la póliza, se hace necesario el uso de mecanismos jurídicos, tales como las acciones civiles y de forma extraordinaria la acción de tutela, que obliguen a las aseguradoras 0 a los centros hospitalarios a que cumplan con su deber legal de proteger a las víctimas de los accidentes de tránsito, bien sea por razón de indemnización o atención medica.

\subsubsection{Incidencia.}

Se entiende por incidencia los efectos que se generan a partir de la relación que se da entre el SOAT y los destinatarios del mismo, de tal manera fue pertinente analizar el grado de conocimiento adquirido con su implementación, la conciencia social en relación del presente fenómeno y finalmente la impresión económica.

Para este fin existe una base estadística derivada de una encuesta aplicada a los adquirientes de la póliza en la ciudad de Neiva. El instrumento que consta de un total de 10 preguntas se aplicó a 701 personas propietarios o conductores de vehículos, que se expone a continuación.

Cuestionario dirigido a propietarios y/o conductores de vehículos:

1. ¿Ha tenido algún tipo de accidente de tránsito?

$\mathrm{Si}$

$$
\text { No }
$$

2. ¿Conoce que es elSOAT?

a) Un seguro de vida.

b) Un seguro de accidentes de tránsito.

c) Unseguro de daño.

d) Un seguro de responsabilidad.

3. ¿Cuál considera que sea la importancia del SOAT?

a) Social, porque cubre los gastos incluso de quienes no tengan seguro medico.

b) Patrimonial, porque cubre gastos generados por el accidente de tránsito.

c) Cultural, porque genera conciencia de transitar con seguro contra accidentes.

d) Todas las anteriores.

4. ¿Cree que la adquisición del SOAT debe ser obligatoria?

a) Si, porque en cualquier momento puede ocurrir un accidente.

b) Si, porque el Estado no puede correr con todos los gastos ocurridos por un accidente.

c) No, porque el costo es muyalto.

d) No, porque estos gastos deberían correr por cuenta del Estado. 
5. ¿Conoce a qué, oa quienes cubre este seguro?

a) Atodos los ocupantes de los vehículos, tanto pasajeros como conductores.

b) Alos peatones involucrados en el accidente.

c) Incluye también reparación a los vehículos.

d) AyB son correctas.

6. ¿Sabecomo reclamar en caso de accidente?

a) Si, porque ya he tenido un accidente yhe tenido que reclamar.

b) Si, porque me he informado acerca del tema.

c) No, porque no existe suficiente información acerca del tema.

d) No, porque no me interesa.

7. ¿Cuáles considera que sean las coberturas de esta póliza?

a) Atención medica por incapacidad o incapacidad permanente.

b) Gastos funerarios e indemnización por muerte.

c) Reparacióna los vehículos.

d) AyBson correctas.

e) Nose.

8. ¿Aqué sanciones cree que se hace acreedor el no portarla póliza o portarla falsa?

a) Comparendo.

b) Comparendoe inmovilización del vehículo.

c) Cancelación de la licencia de conducción y privación de la libertad.

d) Nose.

9. ¿Sabe qué entidad respondeal no portar el SOAT, estar vencido o estar falso en caso de accidente de tránsito?

a) LaEPS a la que este afiliado oel régimen subsidiado.

b) FOSYGA(Fondo de Solidaridad y Garantía)

c) Fondo de prevención vial.

d) Nose.

10. ¿Considera que el costo de la adquisición de esta póliza es excesivo o adecuado?

a) Excesivo, porque el costo de vida es cada vez mas alto.

b) Excesivo, porque tiene muy poca cobertura.

c) Adecuado, porque cubre los gastos necesarios.

d) Adecuado, porque cubre gastos que incluso no deberia cubrir.

A continuación se relacionaran los datos estadísticos arrojados con respecto a seis preguntas encaminadas a determinar el conocimiento básico que se debe tener sobre este seguro: 


\section{Gráfica 1. ¿Conoce que es el SOAT?}

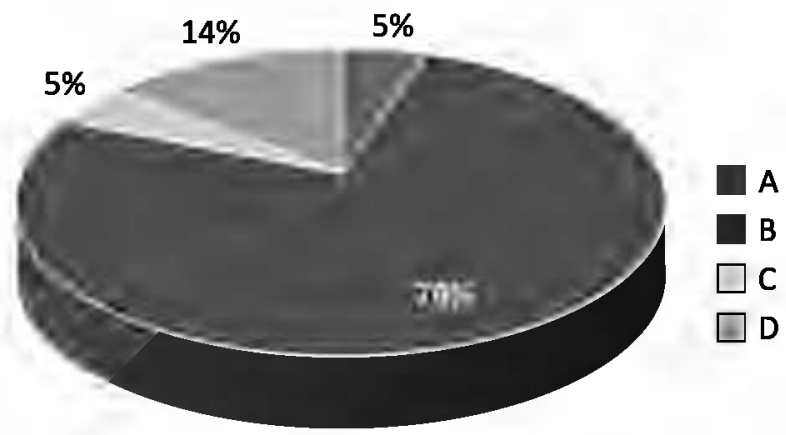
A Un seguro de vida
B Un seguro de accidente de tránsito
C Un seguro de daño
D Un seguro de responsabilidad

- Se observa que respecto a la existencia y naturaleza del seguro las personas poseen un conocimiento significativo al evidenciarse un $76 \%$ del total de la muestra que conoce de su naturaleza jurídica, pero se debe resaltar el $24 \%$ restante de la muestra que confunden el SOAT con otro tipo de seguros que no caben en elámbito del mismo.

\section{Gráfica 2. ¿Conoce a qué, o a quienes cubre este seguro?}

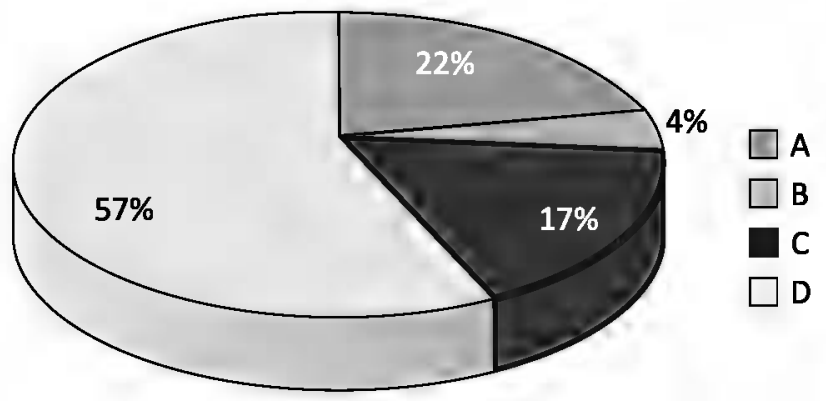

A A todos los ocupantes de los vehículos, tanto pasajeros como conductores

B A los peatones involucrados en el accidente

C Incluye también reparación a los vehículos

D A y B son correctas

- Sobre el ámbito de coberturas un porcentaje bastante representativo $(57 \%)$ conoce a quienes cubre este seguro. Este aspecto resulta importante tenerlo en cuenta, pues a pesar del grado de conocimiento sobre el asunto, un $17 \%$ de la muestra considera que incluso los vehículos entrarían a ser objeto de protección por la póliza, siendo un grave error, pues tal desconocimiento podria conllevar a equívocos al momento del accidente, manifestados en reclamaciones improcedentes, pleitos judiciales sin causa, falsos prejuicios sobre el SOAT, etc.

\section{Gráfica 3. ¿Cuáles considera que sean las coberturas de esta póliza?}

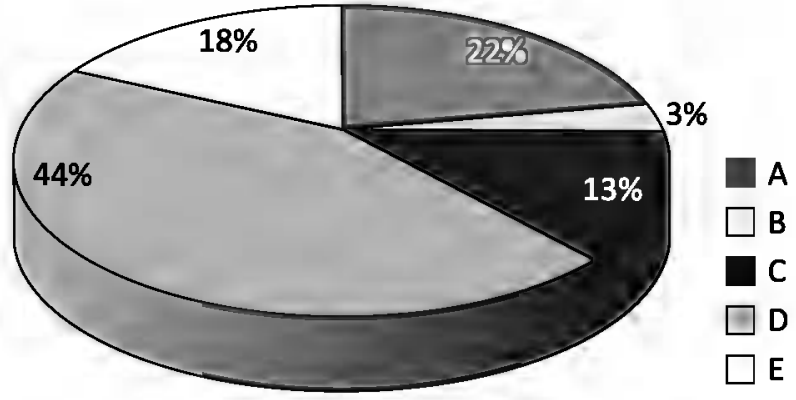

A Atención medica por incapacidad o incapacidad permanente

B Gastos funerarios e indemnización por muerte

C Reparación a los vehículos

D AyB son correctas

E Nose 
- En cuanto a los amparos que han de operar en caso de hacerse exigible la póliza, un total de $56 \%$ no sabe con exactitud las coberturas del seguro. El caso mas singular es el de los gastos funerarios e indemnización por muerte, que en muchas ocasiones las personas del común pasan por alto dejando de recibir tales beneficios.

\section{Gráfica 4. ¿A qué sanciones cree que se hace acreedor el no portar la póliza o portar la falsa?}

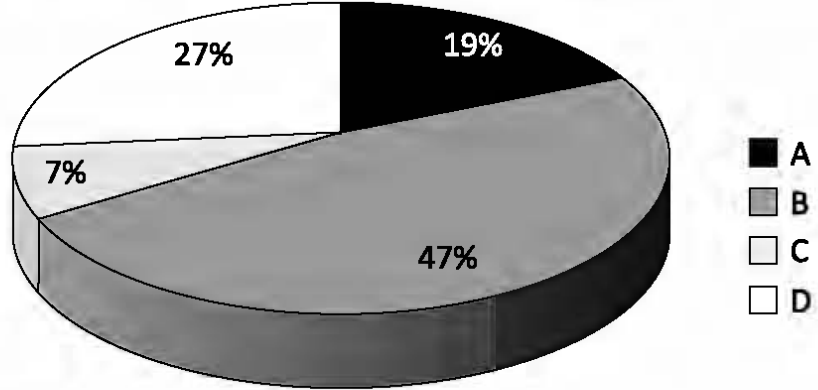
A Comparendo
B Comparendo e inmovilización del vehículo
C Cancelación de la licencia de conducción y privación de la libertad
D Nose

- Sobre las consecuencias jurídicas derivadas de no adquirir este seguro o de portar uno falso un $53 \%$ desconocen las sanciones reales en las que se verian implicados. Este punto es apropiado para quienes conducen vehículos automotores, y resulta preocupante que de las personas encuestadas, 135 crean que solo un comparendo se obtiene como resultado de la inobservancia de las normas de transito, y resulta peor, que 189 encuestados no tengan idea de las sanciones a las que se ven expuestos.

\section{Gráfica 5. ¿Sabe como reclamar en caso de accidente?}

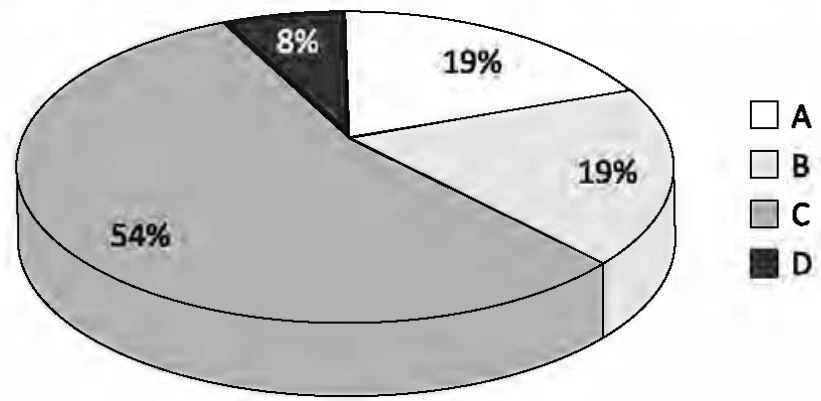
A Si, porque ya he tenido un accidente y he tenido que reclamar
B Si, porque me he informado acerca del tema
C No, porque no existe suficiente información acerca del tema
D No, porque nome interesa

- Otro dato importante que arroja la encuesta tiene que ver con el procedimiento para reclamar y hacer efectivo el cumplimiento de esta póliza, pues el $62 \%$ no sabe como proceder en caso de ocurrir el siniestro. Es preocupantela estadística, pues de ella se desprende el desentendimiento de los tomadores del seguro sobre la importancia de conocer los beneficios del mismo, y desde luego, la manera de acceder a ellos.

Se debe destacar, que un número de 381 encuestados considera que no existe suficiente información acerca del tema. A pesar de entenderse que quien adquiere un seguro debiera interesarse por su conocimiento, debe dejarse claro que este es un Seguro Obligatorio de Accidentes de Transito, y por lo tanto, es el Estado quien debe velar por una comprensión de la pólizaa los tomadores. 
Gráfica 6. ¿Sabe qué entidad responde al no portar el SOAT, estar vencido o estar falso en caso de accidente de transito?

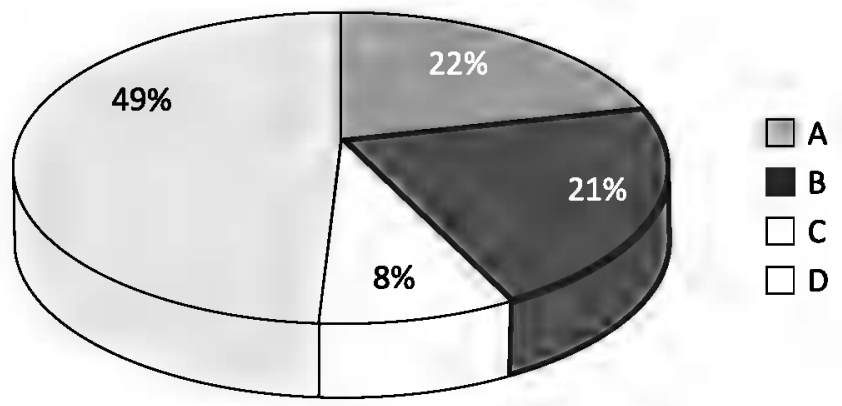
A La EPS a la que este afiliado o el régimen subsidiado
B FOSYGA (Fondo de Solidaridad y Garantía)
C Fondo de prevención vial
D Nose

- Igualmente, se aprecia gran desconocimiento en relación a la autoridad que debe responder en caso que el vehículo no este respaldado por el SOAT 79\%. Esta percepción es errada, y atenta contra los intereses de quienes pudieran concurrir en un accidente de tránsito, porque se debe entender que incluso, si un vehículo se encuentra con el SOAT vencido, falso o no se ha adquirido las víctimas también cuentan con una protección por parte del Estado.

De lo anterior se puede concluir que el conocimiento de las personas encuestadas respecto al SOAT es superficial y en cuanto a aspectos prácticos como las reclamaciones se evidencia una marcada debilidad, que podría ir en detrimento de los intereses económicos de los tomadores del seguro, mas aún, si se analiza que debe adquirirse por obligación.

Con relación al grado de conciencia social se toman como base los siguientes gráficos estadísticos:

\section{Gráfica 7. ¿Cuál considera que sea la importancia del SOAT?}

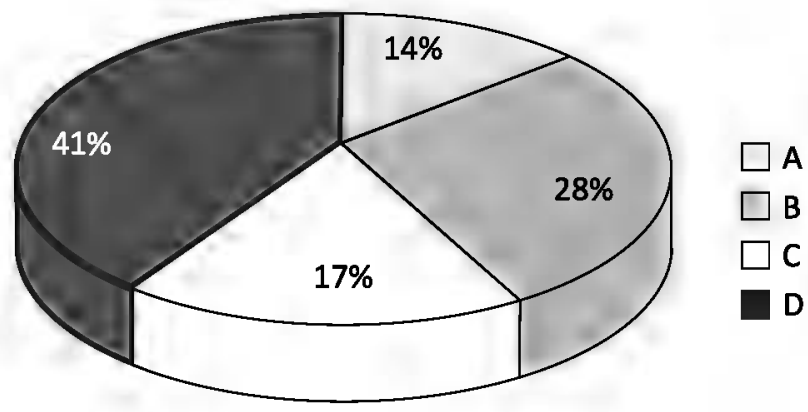
A Social, porque cubre los gastos incluso de quienes no tengan seguro médico
B Patrimonial, porque cubre gastos generados por el accidente de transito
C Cultural, porque genera conciencia de transitar con seguro contra accidentes
D Todas las anteriores

- Se observa que existe una mediana aceptación del seguro, fundamentada en la relevancia social del mismo. Es así como cerca del $41 \%$ de los encuestados reconoce la importancia del SOAT no solo desde su perspectiva económica sino también desde otras esferas como la social y la cultural. Con un $28 \%$, desde luego se percibe el ámbito patrimonial como un elemento de mayor importancia respecto de los otros, por que inevitablemente la adquisición de este seguro toca el bolsillo de los propietarios de vehículos al tener que pagar una prima anual. 


\section{Gráfica 8. ¿Cree que la adquisición del SOAT debe ser obligatoria?}

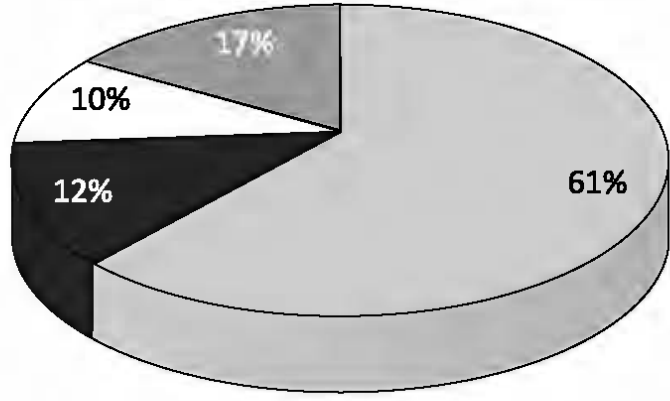

A Si, porque en cualquier momento puede ocurrir un accidente

B Si, porque el Estado no puede correr con todos los gastos ocurridos por un accidente

C No, porque el costo es muy alto

D No, porque estos gastos deberían correr por cuenta del Estado

- Por otro lado, el $61 \%$ de los encuestados consideran que el seguro debe ser obligatorio por la utilidad que presta en caso de un accidente y el $12 \%$ reconoce lo mismo en razón de la limitación del Estado para sufragar la totalidad de los gastos generados por un accidente de tránsito. Este punto suma un total de un $73 \%$ a favor de la obligatoriedad de la póliza, lo cual nos indica un significativo grado de legitimidad del SOAT.

Finalmente, en lo que tiene que ver con la impresión económica del destinatario del seguro se observa el siguiente resultado estadístico:

Gráfica 9. ¿Considera que el costo de la adquisición de esta póliza es excesivo 0 adecuado?

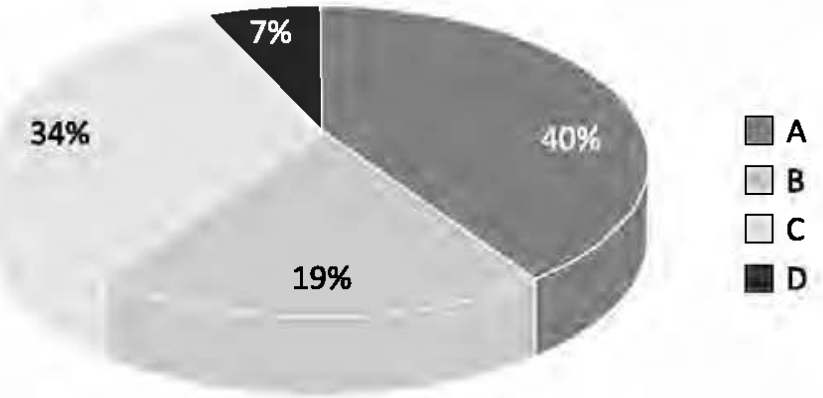

A Excesivo, porque el costo de vida es cada vez mas alto

B Excesivo, porque tiene muy poca cobertura

C Adecuado, porque cubre los gastos necesarios

D Adecuado, porque cubre gastos que incluso no debería cubrir

- En este puntose aprecia que a un $59 \%$ de los encuestados les incomoda el valor de la póliza y el $41 \%$ se encuentra de acuerdo con el valor.Así se advierte que por lo menos la mayoría, aunque la diferencia no es mucha, se encuentra inconforme con la tarifa.

Esta inconformidad para un $40 \%$ se basa en que el costo del seguro les afecta económicamente y para un $19 \%$ en la insuficiencia de la cobertura. Definitivamente la muestra infiere que el pago del SOAT, resulta un gasto que influiría de manera negativa en la economía de los propietarios de vehículos automotores. Esta situación desfavorable puede ser óbice para que los normales tomadores del seguro opten por estrategias más rentables comola no adquisición de la póliza, o peor aún, su falsedad.

De los dos últimos puntos se puede concluir que las personas tienen una mediana conciencia de la importancia de adquirir el SOAT, pero existen factores de tipo económico que podrían desfigurar sus alcances. 
En lo que respecta a la ciudad Neiva se debe tener en cuenta que es una ciudad con un índice de accidentalidad alto respecto de otras ciudades del país con similar número de habitantes según el informe de accidentalidad en las ciudades colombianas (2005-2007) del Fondo de Prevención Vial. $Y$ si se entiende que su principal causa es la masiva circulación de motocicletas, las cuales representan el $50 \%$ del total de vehículos que circulan por el pais que reclaman muertes al SOAT por accidentes de tránsito tal como lo muestra el sistema de información de la cámara técnica del SOAT (FASECOLDA), se hace imprescindible dejar claro que el seguro resulta EFECTIVO en la ciudad, y más aún si se aprecian las cifras en lo que se refiere al costo total que representan los heridos y muertos en los accidentes.

\section{ACCIDENTALIDAD VIAL EN LA CIUDAD DE NEIVA EN EL PERIODO 2005-2007}

\begin{tabular}{lll} 
& NÚMERO & \multicolumn{1}{l}{ COSTO } \\
LEVES & 2427 & $\$ 33.922 .000 .000$ \\
GRAVES & 1809 & $\$ 30.647 .000 .000$ \\
MUERTOS & 175 & $\$ 103.780 .000 .000$ \\
TOTAL & & $\$ 168.349 .000 .000$
\end{tabular}

Definitivamente la gigantesca suma de dinero que se pagó por accidentes de tránsito en la ciudad de Neiva en dos años, demuestra que el SOAT resulta EFECTIVO porque cubre gastos que de otra manera el Estado y las empresas prestadoras del servicio de salud, no serian capaces de sostener.

Sin embargo, Neiva con un parque automotor estimado en 31.847 (por todos tipo de servicio y combustible) según el Ministerio de Transporte, un número total de 58.375 comparendos impuestos en el periodo 2005-2007 por todo tipo de concepto, y comparado con 3.141 comparendos en el mismo periodo solamente por concepto de SOAT (No porte, falsedad, vencimiento), tal como lo indica la Secretaria de Transito Municipal, demuestra que existe una clara falencia en el desarrollo de la
EFECTIVIDAD, que se manifiesta en el incumplimiento de la obligación de adquirir el mismo, quizá por factores mencionados atrás, o por falta de una mayor y estricta exigencia del mismo por parte de las autoridades encargadas.

De esta forma se precisa sobre la toma de conciencia por parte de la población implicada sobre el entendimiento del riesgo que supone el desacatamiento y desconocimiento de la adquisición obligatoria del seguro de accidentes de tránsito.

Al responder la pregunta inicial objeto de la presente investigación, se concluye que el ALCANCE es poco limitado, sin embargo, existen ciertos campos que debieran ser analizados en aras de establecer un mayor ámbito de aplicación del seguro tales como vehículos objetos de protección, pero en especial aquellos evento catalogados como accidentes de tránsito. En este último evento se hace específicamente hincapié a los accidentes que ocurren en lugares sin acceso al público en general.

En cuanto a la EFECTIVIDAD se evalúa que precisamente el Estado cuenta con las herramientas suficientes para lograr la aplicación del SOAT, pero es preciso recalcar la necesidad de una estricta y continua vigilancia para evitar abusos que afecten a los adquirientes del seguro.

Por último, en relación a la INCIDENCIA, a pesar que existe cierto nivel de cultura y conocimiento sobre el SOAT hace falta una aproximación más estrecha entre el seguro y sus destinatarios principalmente en lo que tiene que ver con la relevancia social del mismo y los procedimientos para hacer efectiva la póliza en caso de siniestro. Igualmente, se observa la necesidad de estudiar el fenómeno económico para lograr un equilibrio no desde las perspectivas de las aseguradoras sino desde el destinatario. 


\section{REFERENCIAS BIBLIOGRÁFICAS}

- Conceptos del Comité Juridico de FASECOLDA. (2008). Seguro Obligatorio de Accidentes de Tránsito. Cuarta edición. Bogotá.

- GAVIRIA, Ricardo. (2007). Reglas claras para el proceso de reclamación del SOAT. FASECOLDA, 124, 14-19.

- GAVIRIA, Ricardo. (2008). Accidentes de tránsito se materializan. FASECOLDA, 127,46-51.

- GAVIRIA, Ricardo. (2008). Cambio en las tarifas del SOAT: El ajuste que permite la sostenibilidad financiera del sistema en el país. FASECOLDA, 125, 43-47.

- MARTÍNEZ, Gilberto. (2003). Responsabilidad Civil Extracontractual. Bogotá:Editorial Temis.

- RODRÍGUEZ, Ana Maria. (2008). Análisis económico del seguro obligatorio de accidentes de transito yacuerdos de compensación. Bogotá.

- BAUMEISTER TOLEDO, Alberto (2003). ESTUDIO SOBRE DERECHO DE SEGUROS. Libro homenaje a los 40 años de la Fundación de la Asociación Venezolana de derecho de seguros (AVEDESE-AIDA). Caracas, Universidad CatólicaAndrés Bello.

- BARBATO, Nicolás (2001). Derecho de seguros. Buenos Aires, Editorial Hammurabi.

- STIGLITZ, Rubén (2008). Derecho de seguros. BuenosAires, Editorial La Ley.

- MONTOYA LONDOÑO, Carlos Alberto. Manual de seguros. Bucaramanga, Universidad Autónoma de Bucaramanga.

- SUPERINTENDENCIABANCARIA (1983). El seguro obligatorio de accidentes automoviliarios. Bases para una propuesta. Bogotá, SuperintendenciaBancaria.

- MEILIJ, Gustavo (1975). Tratado de derecho de seguros. BuenosAires, EditoraArgentina.

- DIAZ GRANADOS, Juan Manuel (2006). El seguro de responsabilidad. Bogotá, Editorial Universidad del Rosario. 